\title{
ARTí́CULOS
}

\section{El Clúster de Nanotecnología de Nuevo León: estrategia y operación}

\author{
Jesús González Hernández*
}

\begin{abstract}
Resumen: Se revisa el modelo de cluster como mecanismo para impulsar el desarrollo, entendido como el estímulo a la innovación para el crecimiento económico. Desde ahí se analiza el caso de Nuevo León y, en particular, del avance de la nanotecnología en la región y el impulso del cluster local de nanotecnología. Se presenta una descripción del estado de situación actual y de algunas perspectivas futuras.

Palabras Clave: nanotecnología, innovación, clúster tecnológico, México.

ABSTRACT: The model of clusters is reviewed as a mechanism to promote development, meaning the stimulus of innovation for economic growth. From that starting point it is analyzed the case of the mexican state of Nuevo León. Particularly regarding the development of nanotechnology in the region and the promotion of the local nanotechnology cluster. A description of the current state of the art and some future perspectives are also offered.

KEYWORDS: nanotechnology, innovation, clúster, Mexico.
\end{abstract}

\section{AnTECEDENTES}

El estado de Nuevo León y su ciudad capital Monterrey se han caracterizado históricamente por el espíritu emprendedor de sus habitantes, la calidad de sus instituciones académicas y la competitividad de su sector productivo. Desde principios del siglo pasado, el despliegue industrial de la región ha sido notorio en sectores tales como acero, vidrio, cemento, materiales cerámicos, productos químicos y manufactura metal-mecánica entre otros. Sin embargo, la globalización, la apertura comercial y la celebración de tratados de libre comercio con múltiples países han reconfigurado el entorno competitivo que afecta a la industria y a la sociedad de Nuevo León en general. La competencia es ahora global y las bases de la competitividad y sustentabilidad están hoy altamente relacionadas con la capacidad de una región de adoptar y desarrollar nuevas tecnologías y de crear ecosistemas que privilegien la transferencia de conocimiento.

Es claro que la competitividad de Monterrey está directamente relacionada con su capacidad de atraer, retener y desarrollar oportunidades de inversión y talento estratégico, que en conjunto permitan producir bienes de alto valor agregado y con un componente importante de innovación. Por tal motivo, se crea el Programa Monterrey Ciudad Internacional del Conocimiento (MTYCIC), la que ha privilegiado con énfasis el fortalecimiento de áreas estratégicas de conocimiento que en el mundo desarrollado están transformando a los sectores productivos, destacando: tecnologías de información, mecatrónica, biotecnología, nanotecnología y ciencias de la salud.

\footnotetext{
* Director general del CIMAV; presidente del Cluster de Nanotecnología del Estado de Nuevo León.
} 
Dos de los ejes estratégicos más importantes del Programa MTYCIC son, por un lado, la creación del primer Parque de Investigación e Innovación Tecnológica (PIIT) de Nuevo León, primero en América Latina en integrar 30 organizaciones en un mismo espacio físico (70 hectáreas), entre universidades, empresas y centros de investigación públicos y privados. Se han invertido alrededor de $\$ 1,000$ millones de pesos, y en los próximos años se sumarán aportaciones públicas y privadas que superarán los $\$ 2,000$ millones de pesos. Y, por otro, el impulso de la «coopetencia»-que significa colaboración en la competencia- para establecer clústeres en las áreas estratégicas de conocimiento mencionadas, los cuales también dan una muy alta prioridad a la formación de capital intelectual y a las prácticas de innovación tecnológica.

\section{Clústeres industriales COMO MOdelo de deSARRollo eConómico}

Un clúster en un contexto industrial (o clúster industrial) es una concentración de empresas relacionadas entre sí, en una zona geográfica relativamente definida, de modo de conformar en sí misma un polo productivo especializado con ventajas competitivas. Este concepto fue popularizado por el economista Michael Porter en su libro The competitive advantage of nations (The Free Press, 1990). ${ }^{1}$

Los clústeres en Nuevo León funcionan bajo el modelo conceptual de la Triple Hélice, que implica una alianza entre empresas, universidades y gobierno con el propósito de generar crecimiento económico vía la innovación, beneficiando a la sociedad en su calidad de vida. En el modelo de la Triple Hélice los tres actores principales en el desarrollo de la región se convierten en socios estratégicos para generar un proceso interactivo y sistémico: a) la academia, que, a través de las universidades y centros públicos de investigación, actúa como generadora y propagadora de conocimiento, adicionalmente, el modelo promueve que los investigadores académicos, si lo desean, se conviertan en emprendedores de sus propias innovaciones, invenciones y tecnologías, o que desarrollen alianzas con el sector privado; b) el sector productivo, por su parte, vinculado con el sector académico, utiliza el conocimiento generado para explorar nuevas oportunidades de negocio y realiza también actividades propias de $\mathrm{I}+\mathrm{D}+\mathrm{i}$ a través de inversiones u otro tipo de acciones encaminadas a mejorar su competitividad a través de la aplicación de la innovación y la tecnología en sus productos y servicios; c) el gobierno, finalmente, quien asume un rol activo en el diseño y aplicación de políticas públicas para apoyar la innovación, con lo que proporciona el marco institucional y legal apropiado y genera apoyos e incentivos que permiten tener un entorno y condiciones de crecimiento que impulsan a la región hacia un desarrollo progresivo y sustentable.

A través de la Secretaría de Desarrollo Económico de Nuevo León (SEDEC), se impulsó entonces la integración de los primeros clústeres en los sectores: aeroespacial; automotriz y autopartes; electrodomésticos; tecnologías de información y software; ciencias de la salud y biotecnología. Posteriormente, fueron estructurados e inicializados clústeres en los sectores agroalimentario, nuevos medios digitales y nanotecnología. La conformación de estos primeros clústeres, bajo la participación responsable y

\footnotetext{
1 Para mayores referencias, léase: 1) Sölvell, Örjan. Clusters. Balancing Evolutionary and Constructive Forces. Estocolmo, Suecia. 2008. 2) Sölvell, Örjan; Lindqvist, Göran; Ketels, Christian. The Cluster Initiative Greenbook. Suecia, 2003.
} 
conjunta del sector productivo y la sociedad civil, fue un paso importante para el desarrollo y la consolidación de Nuevo León hacia la economía del conocimiento.

\section{Desarrollo de la nanotecnología en Nuevo León}

La nanotecnología constituye hoy uno de los temas de mayor relevancia científica en el ámbito internacional, con impacto, entre otros, en la medicina, los materiales avanzados, la energía, la electrónica y el medio ambiente. De acuerdo con cifras de Lux Research Inc. (la casa más importante de inteligencia competitiva en nanotecnología), se calcula que actualmente existen a nivel mundial, más de 2,500 compañías involucradas en la nanotecnología, las cuales, ya en el 2006, habían obtenido ingresos del orden de 50,000 millones de dólares anuales, cantidad que, según la proyección más conservadora, crecerá a 250 mil millones en los próximos 10 años. También en el 2006, la inversión mundial en investigación y desarrollo ya alcanzaba los 12,400 millones de dólares, siendo EUA, Japón, Alemania y Corea del Sur los líderes en este rubro, con China siguiendo sus pasos muy de cerca.

En México existe un amplio potencial para los nanomateriales y los nanointermediarios (bienes intermedios construidos con nanomateriales). Se estima que en los próximos 10 años se puedan alcanzar ventas por $\$ 1,500$ millones de dólares, datos basados en un estudio de mercado realizado por la Secretaría de Economía en el año 2008. En ese mismo año, en un primer estudio y análisis del mercado de la región norte, en particular del Estado de Nuevo León, ya se habían podido detectar al menos 25 empresas interesadas en participar en proyectos de nanotecnología .

Una de las razones para la creación del Clúster de Nanotecnología de Nuevo León fue la difusión y el acercamiento de centros de investigación a empresas con proyectos para acelerar su desarrollo y realizar investigación que permita dar solución a los problemas que plantea el mercado. Como un ejemplo de este tipo de esfuerzos, se desarrolló la instalación de la Incubadora de Nanotecnología (miembro y componente esencial del Clúster) con plantas piloto donde se pueden desarrollar y fabricar en cantidades mayores productos que pueden ser utilizados por las empresas de Nuevo León en sus diferentes aplicaciones, permitiendo que se puedan potenciar los proyectos y llevarlos a una escala previa a la industrial. La incubadora es sustentada y dirigida científica y tecnológicamente por el Centro de Investigación en Materiales Avanzados, S.C. (Cimav) del Conacyt.

Es en este contexto de interés en la nanotecnología, y enfoque de recursos y esfuerzos, que opera al día de hoy el Clúster de Nanotecnología, integrado ya por 28 miembros (arrancó con 15 miembros). Está integrado por 3 centros públicos de investigación, 3 universidades mexicanas, 2 universidades extranjeras, 2 instituciones de gobierno (SEDEC e I ${ }^{2} \mathrm{~T}^{2}$ ), y 18 empresas, 2 de ellas Pymes. De los anteriores, destacan el Cimav (Centro de Investigación en Materiales Avanzados del Conacyt); el Tecnológico de Monterrey; la Universidad Autónoma de Nuevo León, las universidades estadounidenses de Arizona y de Texas en Austin; y empresas de los corporativos regios CEMEX, ALFA, VITRO, CYDSA Y PROEZA.

Particularmente el Cimav, cuya sede está en Chihuahua, se ha convertido en uno de los actores más importantes en el desarrollo de aplicaciones industriales de la nanotecnología. En el 2010, sólo este centro de investigación tenía vigentes cerca de 25 proyectos con empresas de la región en temas de nanotecnología. 


\section{Estrategia central del Clúster de Nanotecnología de Nuevo León}

El Clúster tiene como objetivos de origen el desarrollo de capital humano experto, la atracción de financiamiento y la implementación de proyectos de nuevos negocios con materiales o aplicaciones basados o relacionados con la nanotecnología. Siendo su misión la adopción de la nanotecnología en Nuevo León, su visión consiste, en términos generales, en crear las condiciones necesarias para que al menos 100 empresas adopten la nanotecnología dentro de sus desarrollos para el año 2015. Se busca también constituir el Sistema Regional de Innovación en Nanotecnología más importante del país, propiciando la transferencia de tecnología y conocimiento entre los miembros de su red de asociados y consecuentemente hacia la mayor cantidad posible de empresas de la región. Se busca también, colaborar en el desarrollo económico del Estado mediante el apoyo a empresas Pymes, catalizando su inserción en mercados de oportunidad internacionales.

El Clúster de Nanotecnología de Nuevo León tiene entonces dos vertientes estratégicas de igual importancia: a) el desarrollo y mantenimiento de un pipeline de nanotecnología mediante proyectos de innovación incremental entre los grandes grupos industriales que lo conforman y mediante metodologías de innovación abierta, y b) la creación de nuevas empresas de base tecnológica, con proyectos de innovación de naturaleza mas disruptiva, pero de gran importancia en la generación de tecnología mexicana y en el enriquecimiento de las cadenas de valor industriales existentes. Sin dejar de mencionar la muy alta relación entre la incidencia de nuevas empresas de naturaleza tecnológica emergente y el crecimiento del ingreso per cápita de cualquier región.

\section{MODELO DE OPERACIÓN}

Para consolidar la ejecución de la Estrategia Central del Clúster de Nanotecnología de Nuevo León, su operación se ha dividido en 4 comités de trabajo, cada uno de ellos presidido por importantes miembros de la sociedad de Nuevo León y de su planta productiva.

Comité de Innovación y Transferencia de Tecnología. Presidido por el Dr. Salvador Valtierra, director de Innovación para América Latina de la trasnacional Owens Corning. Los principales proyectos de este Comité consisten en: a) el establecimiento de un sistema de administración para el pipeline del Clúster, fundamentalmente basado en las metodologías Stage Gate para la administración del desarrollo de nuevos productos y, b) la estructuración de un sistema de vigilancia tecnológica que permita a los asociados del Clúster monitorear aspectos relevantes de la nanotecnología, tanto bajo la perspectiva científica como bajo la de negocios.

Comité de Estrategia y Expansión. Presidido por el Ing. Alfonso Delgado, miembro de la alta dirección de PROLEC GE en Nuevo León. Los principales proyectos de este Comité consisten en: a) el desarrollo del plan estratégico a 5 años del Clúster, incluyendo prospectiva y mapas tecnológicos, actualmente en proceso; b) el desarrollo de un modelo de valor/aplicación para cada uno de los sectores industriales más relevantes en el sector productivo de Nuevo León, enfocado a la resolución de problemas específicos a nivel materiales de producción y procesos, actualmente en proceso; c) el establecimiento de una plataforma web de colaboración entre los asociados miem- 
bros del Clúster, en una temática tipo hub colaborativo de innovación abierta y, d) la constitución de la imagen institucional del Clúster.

Comité de Desarrollo de Talento Estratégico. Presidido por el Ing. Luis Cabeza del Tecnológico de Monterrey, Campus Monterrey. Los principales proyectos de este Comité consisten en: a) el desarrollo de programas industriales de capacitación en nanotecnología para los miembros del Clúster y, b) el desarrollo de posgrados interinstitucionales en nanotecnología en el cual participen los asociados académicos del mismo.

Comité de Financiamiento y Desarrollo de Nuevos Negocios. Presidido por el C.P. Federico García Iza, importante miembro de la comunidad empresarial de Nuevo León en el rubro de inversiones y capital privado. El principal proyecto de este Comité consiste en la institucionalización de mecanismos de inversión privada para nuevas empresas de base tecnológica. Los formatos actualmente en proceso de desarrollo contienen elementos de los tradicionales grupos de inversionistas "Angeles" pero la infraestructura y formatos de la industria de capital de riesgo o venture capital. Actualmente se le está dando forma a estos mecanismos y se están explorando los lineamientos legales a seguir.

\section{CONCLUSIONES Y PERSPECTIVAS FUTURAS}

A casi dos años de su creación, el Clúster de nanotecnología de Nuevo León se está consolidando como una nueva opción de crecimiento y competitividad para las empresas locales y promotor insignia de un nuevo modelo de desarrollo para el Estado de Nuevo León. En el contexto competitivo global, existe la urgencia de actualizar la industria de la transformación de Nuevo León, con la incorporación de nuevas tecnologías, sobre todo en el uso de materiales avanzados, que en muchos países desarrollados ya representan el estándar.

Se espera que el Clúster de nanotecnología de Nuevo León permita consolidar su ventaja competitiva al precisamente generar elementos de competitividad especializado a sus asociados miembros y detonar el surgimiento de nuevas empresas y negocios de base tecnológica.

El Clúster es un elemento muy importante del Programa Monterrey Ciudad Internacional del Conocimiento, es una gran alianza entre la academia, el sector productivo y gobierno para detonar una economía en la cual se crea, se transmite, se adquiere y se utiliza el conocimiento con mayor efectividad por sus ciudadanos y sus organizaciones, con el objetivo de promover el desarrollo económico y social de la propia comunidad basado en la innovación. 